\title{
Plasma gonadotrophin and ovarian steroid concentrations in women with menstrual cycles with a short luteal phase
}

\author{
S. K. Smith*, Elizabeth A. Lenton and I. D. Cooke \\ University Department of Obstetrics and Gynaecology, Jessop Hospital for Women, Leavygreave Road, \\ Sheffield S3 7RE, U.K.
}

\begin{abstract}
Summary. Daily plasma concentrations of FSH, LH, oestradiol-17 $\beta$ and progesterone were compared for 12 cycles with a short luteal phase and 19 cycles with a luteal phase of normal length (i.e. cycles in which the luteal phase lasted 12 or more days). FSH and LH concentrations were suppressed in short luteal-phase cycles in the early follicular phase and the length of the follicular phase was prolonged (median duration, 14.5 days, range 13-21 days: compared with 12 days, range 9-17, in control cycles; $P<0.025$ ). Preovulatory oestradiol-17 $\beta$ values and the mid-cycle concentrations of FSH and LH were similar in both groups. Plasma progesterone values in the luteal phase were similar in both groups over the 2 nd to 5 th days inclusive after the midcycle LH peak but declined in the short luteal phases thereafter. In short luteal-phase cycles, menstruation occurred in the presence of higher levels of oestradiol-17 $\beta$ and progesterone than in cycles of normal length and the rise of gonadotrophin in the late luteal phase of the cycle was delayed. These findings suggest that in cycles with a short luteal phase there is a lack of synchrony between the ovarian and menstrual events.
\end{abstract}

\section{Introduction}

The interval between menstrual bleeding in women is determined by the lengths of the follicular and luteal phases of the cycle. Follicular-phase length follows a log-normal distribution but lutealphase length demonstrates a bimodal distribution, the lower $95 \%$ confidence limit being 12 days (Lenton, Landgren \& Sexton, 1984a; Lenton, Landgren, Sexton \& Harper, 1984b). Cycles in which the luteal phase lasts $<11$ days are recognized as part of a spectrum of abnormalities of corpus luteum function (Jones, 1976; DiZerega \& Hodgen, 1981).

In rhesus monkey and human females, corpus luteum function is influenced by events in the follicular phase of the cycle. Suppression of blood FSH concentrations at the beginning of the cycle by the administration of pig follicular fluid to monkeys causes a reduction of progesterone concentrations in the luteal phase and may induce cycles with a short luteal phase (Stouffer \& Hodgen, 1980 ). Concurrent administration of human menopausal gonadotrophin results in partial compensation of corpus luteum function and cycles with a luteal phase of nearly normal length. Previous studies in women who have cycles with a short luteal phase have shown depressed plasma levels of progesterone in the luteal phase, poor follicular development and subnormal plasma concentrations of FSH at the beginning of the cycle (Strott, Cargille, Ross \& Lipsett, 1970; Sherman \& Korenman, 1974).

The aim of this study was to determine the relationship between gonadotrophin concentrations in the early follicular phase of the cycle in women with cycles that had a short or normal luteal phase length and to compare the subsequent development of the follicle and the function of the corpus luteum.

* Present address: MRC Reproductive Biology Unit, Centre for Reproductive Biology, 37 Chalmers Street, Edinburgh EH3 9EW, U.K. 


\section{Materials and Methods}

Daily venous blood samples were obtained from women during the course of a larger study of daily hormone profiles. Blood was centrifuged and plasma stored at $-20^{\circ} \mathrm{C}$. Plasma concentrations of oestradiol-17 $\beta$ and progesterone were measured by radioimmunoassays as described previously (Lenton, Adams \& Cooke, 1978). The antisera were kindly provided by Dr R. Scaramuzzi, Edinburgh, and Dr B. Furr. Macclesfield. The inter- and intra-assay coefficients of variation for the assay of oestradiol-17 $\beta$ were 12.9 and $5.2 \%$ and for progesterone 8.8 and $7.0 \%$ respectively. The sensitivities of the assays, being the least concentration of hormone that was consistently detectable from zero, were $40 \mathrm{pmol}$ oestradiol $/ \mathrm{l}$ and $0.6 \mathrm{nmol}$ progesterone $/ 1$.

Plasma FSH and LH concentrations were measured by double-antibody radioimmunoassays and the results are expressed as $\mathrm{u} / \mathrm{l}$ in terms of the 2 nd International Reference Preparation of Human Menopausal Gonadotrophin (78/549 and 68/40) kindly provided by the World Health Organization from the National Institute for Biological Standards, London. The inter- and intra-assay coefficients of variation for the assay of FSH were 11.3 and $6.1 \%$ and for $\mathrm{LH} 10.3$ and $5 \cdot 2 \%$ respectively. The sensitivities of the assays were $1.5 \mathrm{u} \mathrm{FSH} / 1$ and $4 \mathrm{u} \mathrm{LH} / 1$.

\section{Results}

Plasma concentrations of FSH in the cycles with short luteal phases were lower than those in women with normal cyles over the first 3 days of the cycle $(P<0 \cdot 05)$, but rose to reach control values by Day 4 of the cycle (Text-fig. 1). In individual short luteal-phase cycles, the median day of the highest value obtained for plasma FSH was Day 6 (range 3-11 days) compared with Day 3 (range $1-9$ days) in the control cycles $(P<0.005)$. LH values were lower on Day 1 of the cycle in women with short luteal-phase cycles but were similar to values in the control group by Day 2 .

As a consequence of the delayed rise in plasma FSH concentrations in the short luteal-phase cycles, follicular development, as judged by the rise of peripheral concentrations of oestradiol-17 $\beta$, was delayed by 3-5 days (Text-fig. 1). However, the time from the highest level of FSH in individual cycles to the mid-cycle LH peak was similar in both groups (short luteal-phase cycles: median, Day 8, range 5-17 days; control cycles: median Day 9, range 5-13 days). Follicular development was not impaired in women with short luteal-phase cycles, as preovulatory plasma oestradiol-17 $\beta$ values were comparable with those found in control cycles, when the hormonal data were centred on the day of the maximum LH concentration in plasma (see Text-fig. 2). Mid-cycle plasma concentrations of FSH and LH were similar in both groups.

In the luteal phase, plasma progesterone concentrations were similar in both groups until the 6th day after the LH peak, but declined thereafter in the cycles with a short luteal phase. Despite this, not all of the cycles in which the luteal phase was reduced had poor function of the corpus luteum. The average concentrations of progesterone in plasma on the 2 nd to 5 th day inclusive after the mid-cycle LH peak were similar in both groups. Even over the 5th to 8 th days inclusive after the LH peak, 7 of the short luteal-phase cycles had average progesterone concentrations within 1 standard deviation of the mean of the average levels of the control group.

A possible explanation for the delayed rise of gonadotrophin concentrations in the study cycles is provided by examination of the hormone profiles in the late luteal phase of the cycle. Plasma concentrations of oestradiol-17 $\beta$ were consistently higher in cycles with a short luteal phase than in control cycles in the 6 days before the onset of menstrual bleeding (Text-fig. 1). Conversely, FSH and LH concentrations in plasma were depressed in the late luteal phase of the cycle and the rise of gonadotrophin values seen in control cycles was delayed in the cycles with a short luteal phase. In these premenstrual days, plasma progesterone concentrations were lower in the short luteal-phase cycles compared to control cycles until the 2 nd day before menstruation, but were higher $24 \mathrm{~h}$ before the onset of menstruation (Text-fig. 1) $(P<0.05)$. 


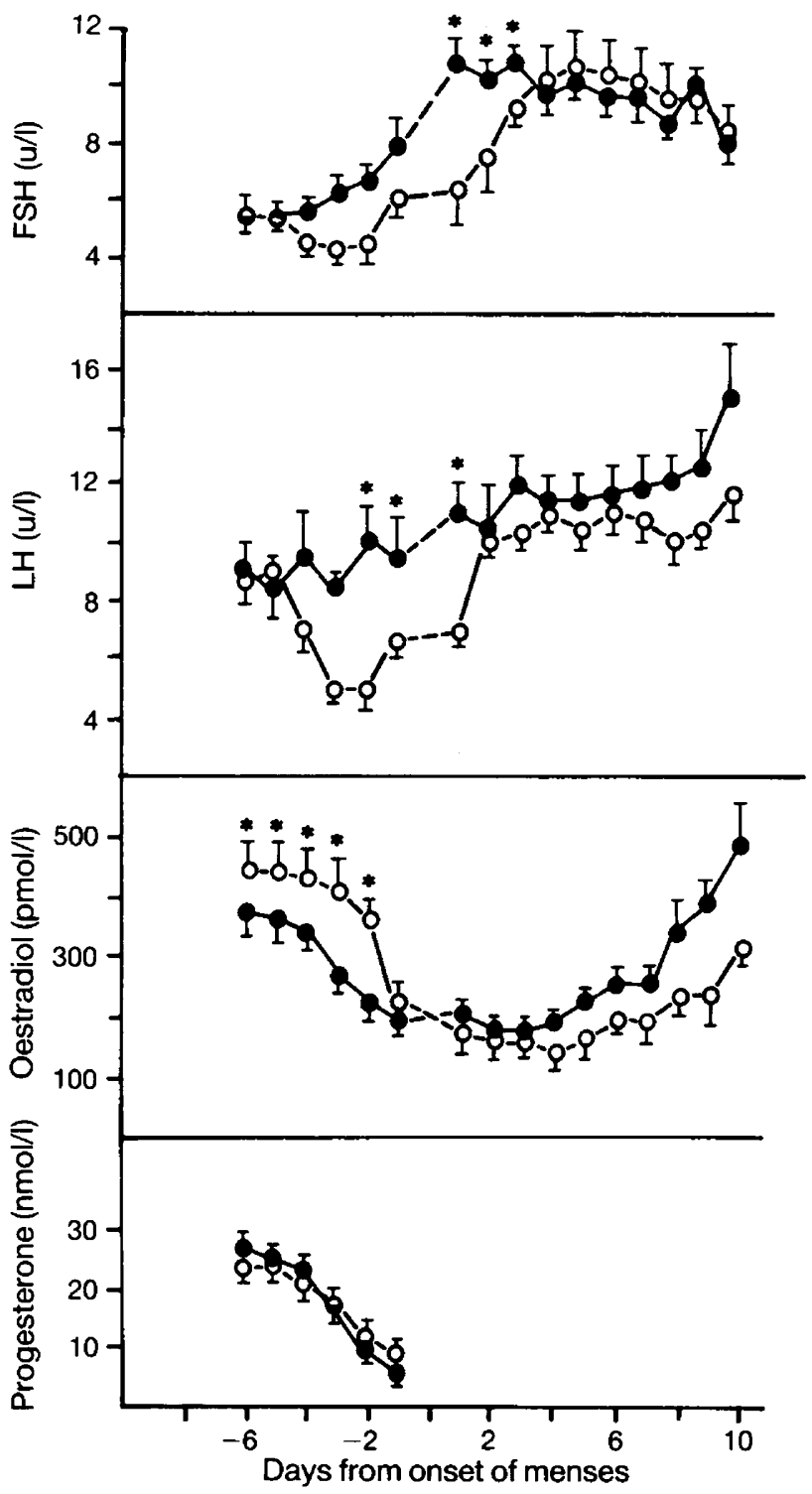

Text-fig. 1. Mean \pm s.e.m. plasma concentrations of FSH, LH, oestradiol-17 $\beta$ and progesterone in short luteal-phase and control cycles in women before and after the onset of menstruation. ${ }^{*} P=0.05$.

\section{Discussion}

The findings from this study indicate that plasma gonadotrophin concentrations in cycles with a short luteal phase were suppressed at the time of menstruation. FSH levels then rose to reach their highest values on the 5th day of the cycle and, although follicular development was delayed, it was not impaired as judged by the measurement of oestradiol-17 $\beta$ concentrations in plasma. Concentrations of $\mathrm{LH}$ rose more quickly than those of FSH and were similar to control values by the 


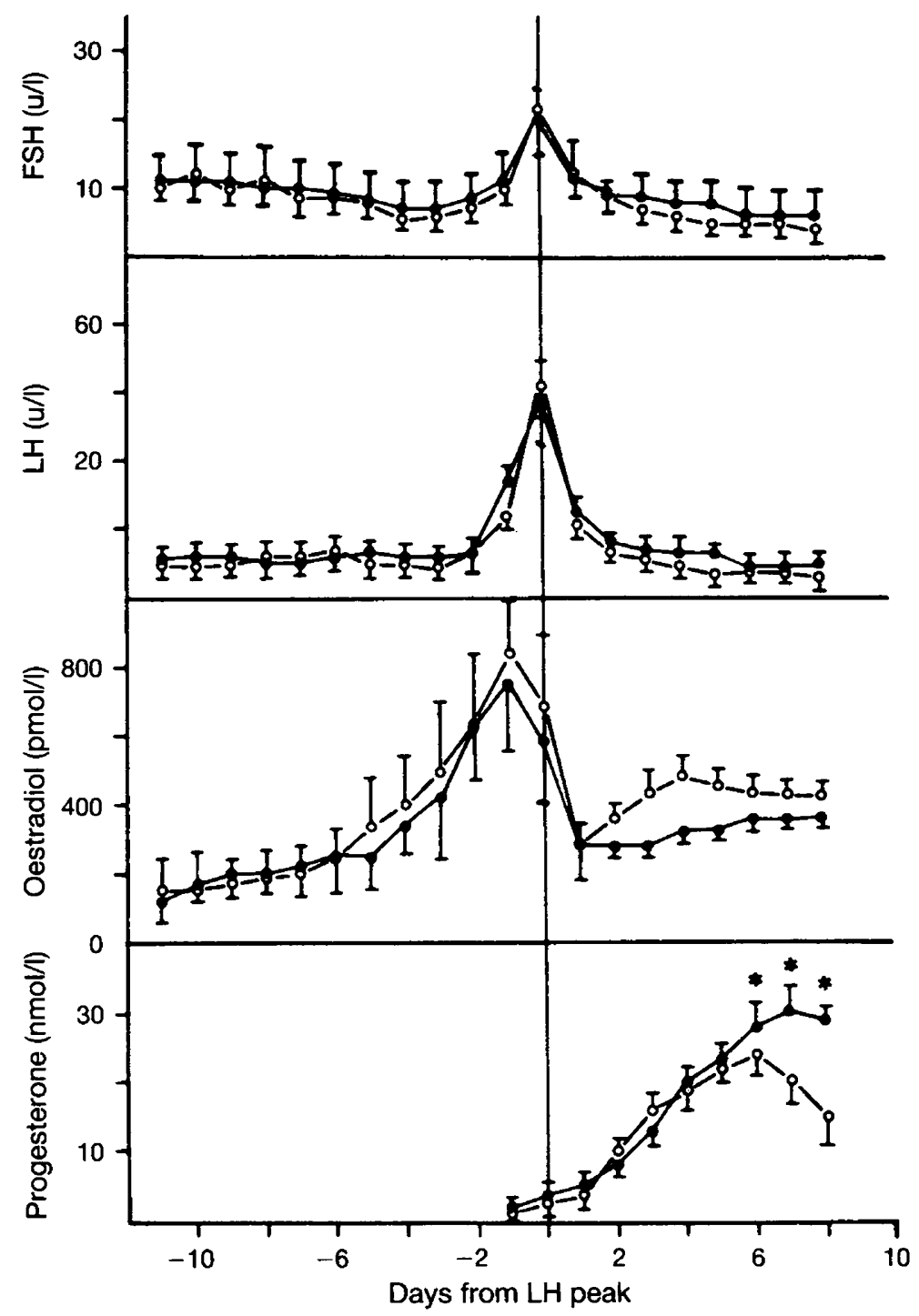

Text-fig. 2. Mean \pm s.e.m. plasma concentrations of FSH, LH, oestradiol-17 $\beta$ and progesterone in short luteal-phase and control cycles when data are entered on the mid-cycle LH surge. ${ }^{*} P=0.05$.

2nd day of the cycle. The suppression of gonadotrophin concentrations at the beginning of the cycle may reflect a delayed rise of plasma FSH and LH concentrations in the late luteal phase because of the enhanced negative feedback effect of the higher plasma values of oestradiol-17 $\beta$ in the premenstrual period.

In cycles of normal length, plasma concentrations of FSH and LH begin to rise in the late-luteal phase as a consequence of regression of the corpus luteum (Ross et al., 1970). Enucleation of the corpus luteum in women is followed by a rapid decline in the plasma oestradiol-17 $\beta$ and progesterone values (Aedo, Pedersen, Pedersen \& Diczfalusy, 1980; Nilsson, Wikland \& Hamberger, 
Table 1. Median (and range) length of the follicular and luteal phase in cycles with a short or normal luteal-phase length in women

\begin{tabular}{lccc}
\hline & \multicolumn{3}{c}{ Menstrual cycle length (days) } \\
\cline { 2 - 4 } Luteal phase & Follicular & Luteal & Total \\
\hline Short & 15 & 11 & 27 \\
& $(11-20)$ & $(8-11)$ & $(23-29)$ \\
Normal & 12 & 14 & 27 \\
& $(9-17)$ & $(12-15)$ & $(24-32)$ \\
\hline
\end{tabular}

1982; Baird, Backström, McNeilly, Smith \& Wathen, 1984) and resumption of follicular activity follows a rise in the concentrations of FSH. The prolonged follicular phase in short luteal-phase cycles (Table 1) could have arisen because of the delay in the rise of FSH, which is similar to the findings of Baird et al. (1984) after removal of the corpus luteum.

It was not possible from this study to determine whether the delay of the follicular development arose because of the systematic inhibition of gonadotrophin levels by oestradiol-17 $\beta$ and/or eventually progesterone (Faiman \& Ryan, 1967; Baird, Baker, McNatty \& Neal, 1975) or whether progesterone itself exerted a local block on the development of non-atretic follicles (DiZerega \& Hodgen, 1981).

A further possibility is that differences in the concentrations of other factors, such as inhibin (Stouffer \& Hodgen, 1980), could have been responsible for the subnormal FSH values, as apart from the first day of the follicular phase there was selective suppression of FSH concentrations in comparison to those of $\mathrm{LH}$.

The findings from this study cannot be reconciled with the view that short luteal-phase cycles always show poor or defective corpus luteum function and that they follow poor follicular development. Low plasma progesterone concentrations in the luteal phase of the cycle were found more frequently in cycles with a short luteal phase than in cycles of normal length but, despite this, 7 of the 12 cycles had plasma progesterone values over the 5th to 8th days after the LH peak that were within one standard deviation of the values in control cycles. Follicular development was delayed in the study cycles, but subsequent levels of oestradiol-17 $\beta$ in the preovulatory period were similar in both groups. This failure to confirm the findings of Strott et al. (1970) and Sherman \& Korenman (1974) in which short luteal-phase cycles followed poor follicular function is, however, consistent with the observations of DiZerega, Stouffer \& Hodgen (1981) who showed that proliferation of granulosa cells was not impaired when porcine follicular fluid was used to cause a transient reduction of plasma FSH levels at the beginning of the primate cycle. Further, the number of dispersed luteal cells present in the corpora lutea of monkeys is not reduced in those animals that have defective corpus luteum function. These findings suggest that prolongation of the follicular phase found in the cycles with a short luteal phase, as reported here, may not have impaired the development of the granulosa cells but that the reduction of the length of the luteal phase may be a consequence of abnormal differentiation of granulosa cells or a disorder of LH/FSH-dependent steroidogenesis (DiZerega \& Hodgen, 1981).

Menstruation in primates follows the withdrawal of progesterone from an oestrogen-primed endometrium (Corner, 1963). However, the early onset of menstrual bleeding in the cycles with a short luteal phase occurs in the presence of higher levels of oestradiol-17 $\beta$ and progesterone than in cycles of normal luteal-phase length. These findings suggest that cycles with a short luteal phase may not only reflect disorders of corpus luteum function but also a premature response of the endometrium to declining concentrations of ovarian steroids. 


\section{References}

Aedo, A.R., Pedersen, P.H., Pedersen, S.C. \& Diczfalusy, E. (1980) Ovarian steroid secretion in normally menstruating women II. The contribution of the corpus luteum. Acta endocr., Copenh. 95, 222-231.

Baird, D.T., Baker, T.G., McNatty, K.P. \& Neal, P. (1975) Relationships between the secretion of the corpus luteum and the length of the follicular phase of the ovarian cycle. J. Reprod. Fert. 45, 611-619.

Baird, D.T., Backström, T., McNeilly, A.S., Smith, S.K. $\&$ Wathen, C.G. (1984) Effect of enucleation of the corpus luteum at different stages of the luteal phase of the human menstrual cycle on subsequent follicular development. J. Reprod. Fert. 70, 615-624.

Corner, G.W. (1963) The Hormones in Human Reproduction. Atheneum, New York.

DiZerega, G.S. \& Hodgen, G.D. (1981) Luteal phase dysfunction infertility: a sequel to aberrant folliculogenesis. Fert. Steril. 35, 489-499.

DiZerega, G.S., Stouffer, R.L. \& Hodgen, G.D. (1981) FSH regulation of intrafollicular events in the primate ovarian cycle. In Intragonadal Regulation of Reproduction, pp. 9-31. Eds C. P. Channing \& P. Franchimont. Academic Press, New York.

Faiman, C. \& Ryan, R.J. (1967) Serum follicle-stimulating hormone and luteinizing hormone concentrations during the menstrual cycle as determined by radioimmunoassays. J. clin. Endocr. Metab. 27, 1711-1716.

Jones, G.S. (1976) The luteal phase defect. Fert. Steril. 27, 351-356

Lenton, E.A., Adams, M. \& Cooke, I.D. (1978) Plasma steroid and gonadotrophin profiles in ovulatory but infertile women. Clin. Endocr. 8, 241-255.
Lenton, E.A., Landgren, B.M., Sexton, L. (1984a) Normal variation in the length of the luteal phase of the menstrual cycle: identification of the short luteal phase. Br. J. Obstet. Gynaec. 91, 685-689.

Lenton, E.A., Landgren, B.M., Sexton, L. \& Harper, R. (1984b) Normal variation in the length of the follicular phase of the menstrual cycle: effect of chronological age. Br.J. Obstet. Gynaec. 91, 681-684.

Nilsson, L., Wikland, M. \& Hamberger, L. (1982) Recruitment of an ovulatory follicle in the human following follicle-ectomy and lute-ectomy. Fert. Steril. 37, 30-34.

Ross, G.T., Cargille, C.M., Lipsett, M.B., Radford, P.L., Marshall, J.R., Scott, C.A. \& Robbard, D. (1970) Pituitary and gonadal hormones during spontaneous and induced ovulatory cycles. Recent Prog. Horm. Res. 26, 1-62.

Sherman, B.M. \& Korenman, S.G. (1974) Measurement of plasma LH, FSH, oestradiol and progesterone in disorders of the human menstrual cycle: the short luteal phase. J. clin. Endocr. Metab. 38, 89-93.

Siegel, S. (1956) Non-Parametric Statistics. McGrawHill, New York.

Stouffer, R.L. \& Hodgen, G.D. (1980) Induction of luteal phase defects in rhesus monkeys by follicular fluid administration at the onset of the menstrual cycle. $J$. clin. Endocr. Metab. 51, 699.

Strott, C.A., Cargille, C.M., Ross, G.T. \& Lipsett, M.B. (1970) The short luteal phase. J. clin. Endocr. Metab. 30, 246-251.

Received 28 November 1984 